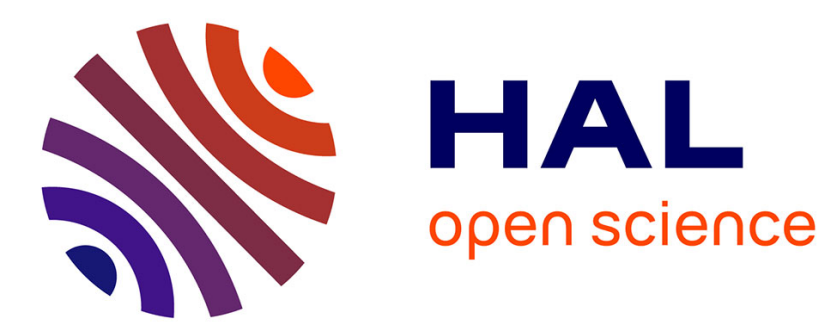

\title{
Conditional Mean-Variance and Mean-Semivariance models in portfolio optimization
}

\author{
Hanene Ben Salah, Ali Gannoun, Mathieu Ribatet
}

\section{To cite this version:}

Hanene Ben Salah, Ali Gannoun, Mathieu Ribatet. Conditional Mean-Variance and MeanSemivariance models in portfolio optimization. 2016. hal-01299566

\section{HAL Id: hal-01299566 https://hal.science/hal-01299566}

Preprint submitted on 12 Apr 2016

HAL is a multi-disciplinary open access archive for the deposit and dissemination of scientific research documents, whether they are published or not. The documents may come from teaching and research institutions in France or abroad, or from public or private research centers.
L'archive ouverte pluridisciplinaire HAL, est destinée au dépôt et à la diffusion de documents scientifiques de niveau recherche, publiés ou non, émanant des établissements d'enseignement et de recherche français ou étrangers, des laboratoires publics ou privés. 


\title{
Towards Dynamical Portfolio allocation Selections
}

\author{
Hanene Ben Salah ${ }^{1,2,3}$, Ali Gannoun ${ }^{3}$, Christian de Peretti ${ }^{2}$, Mathieu Ribatet ${ }^{3}$,
} Abdelwahed Trabelsi ${ }^{1}$

${ }^{1}$ BESTMOD Laboratory, ISG 41 Rue de la Liberté, Cité Bouchoucha 2000 Le Bardo,Tunisie,bensalah_hanen@yahoo.fr, abdel.trabelsi@gmail.com

2 Univ Lyon, Université Claude Bernard Lyon 1, Institut de Science Financière et d'Assurances, LSAF EA2429, F-69366_Lyon, France. ,Christian.De-Peretti@ec-lyon.fr

${ }^{3}$ IMAG34095 Montpellier cedex 05, ali.gannoun@um2.fr, mathieu.ribatet@um2.fr.

Abstract : In this paper, we consider the problem of portfolio optimization. The risk will be measured by conditional variance or semivariance. It is known that the historical returns used to estimate expected ones provide poor guides to future returns. Consequently, the optimal portfolio asset weights are extremely sensitive to the return assumptions used. Getting informations about the future evolution of different asset returns, could help the investors to obtain more efficient portfolio. The solution will be reached under conditional mean estimation and prediction. This strategy allows us to take advantage from returns prediction which will be obtained by nonparametric univariate methods. Prediction step uses kernel estimation of conditional mean. Application on Chinese and American markets are presented and discussed.

Keywords : Conditional Semivariance, Conditional Variance, DownSide Risk, Kernel Method, Nonparametric Mean prediction.

\section{Introduction}

Investment strategies and their profitability have always been a hot topic for people with an interest in financial assets. The modern asset allocation theory was originated from the Mean-Variance portfolio model introduced by Markowitz (1952), see also Markowitz (1959) and Markowitz (1987). The original Markowitz model simply dealt with a static single period asset allocation problem. Variance is commonly used as a risk measure in portfolio optimisation to find the trade-off between the risk and return. Variance measures the deviation above and below the mean return. Investors wish to minimise the risk at the given return level. Variance and mean are estimated with the data observed before the portfolio optimization date.

Markowitz's portfolio optimization requires the knowledge both of the expected return and the covariance matrix of the assets. It is well known that the optimum portfolio weights are very sensitive to return expectations which are very difficult to determine. For instance, historical returns are bad predictors of the future returns (Michaud, 1989; Black and Littermann, 1992 and Sigel, 2007). Estimating covariance matrices is a delicate statistical 
challenge that required sophisticated methods (see Ledoit and Wolf, 2004). It is fair to state that, due to the large statistical errors of the input of Markowitz's portfolio optimization, its result is not reliable and should be considered very cautiously. This led Levy and Roll (2010) to turn the usual approach on its head and found that minor adjustments of the input parameter are needed, well within the statistical uncertainties.

In this paper, we propose radically different perspective by including, in the estimation step, informations about the possible future returns, obtained by nonparametric prediction. Than, we can improve the quality of portfolio optimisation. Firstly, we exhibit the classical Markowitz model developed in 1952.

Let us say that there are $m$ assets to constitute a portfolio $P$ and denote by $r_{j t}$ the return of asset $j$ on date $t, t=1, \ldots, T$, and $M$ the estimated variance-covariance matrix of the returns $\left(r_{1}, \ldots, r_{m}\right)$,

$$
M=\frac{1}{T} \sum_{t=1}^{T}\left[\begin{array}{ccc}
\left(r_{1 t}-\bar{r}_{1}\right)^{2} & \left(r_{1 t}-\bar{r}_{1}\right)\left(r_{2 t}-\bar{r}_{2}\right) & \left(r_{1 t}-\bar{r}_{1}\right)\left(r_{m t}-\bar{r}_{m}\right) \\
\left(r_{2 t}-\bar{r}_{2}\right)\left(r_{1 t}-\bar{r}_{1}\right) & \left(r_{2 t}-\bar{r}_{2}\right)^{2} & \left(r_{2 t}-\bar{r}_{2}\right)\left(r_{m t}-\bar{r}_{m}\right) \\
\vdots & \vdots & \vdots \\
\left(r_{m t}-\bar{r}_{m}\right)\left(r_{1 t}-\bar{r}_{1}\right) & \left(r_{m t}-\bar{r}_{n}\right)\left(r_{2 t}-\bar{r}_{2}\right) & \left(r_{m t}-\bar{r}_{m}\right)^{2}
\end{array}\right] .
$$

The optimization program is then the following

$$
\min _{\omega} \omega^{\top} M \omega \text {, subject to } \omega^{\top} \mu=E^{*}, \omega^{\top} \mathbf{1}=1,
$$

where $\omega^{\top}=\left(\omega_{1}, \ldots, \omega_{m}\right)$ is the portfolio vector weight, $\mu^{\top}=\left(\bar{r}_{1}, \ldots, \bar{r}_{m}\right)=\left(\frac{1}{T} \sum_{t=1}^{T} r_{1 t}, \ldots, \frac{1}{T} \sum_{t=1}^{T} r_{m t}\right)$ the empirical mean returns and $E^{*}$ is a target expected portfolio return.

Using Lagrangian method, the explicit solution of solution of (2) is :

$$
\omega_{*}=\frac{\alpha E^{*}-\lambda}{\alpha \theta-\lambda^{2}} M^{-1} \mu+\frac{\theta-\lambda E^{*}}{\alpha \theta-\lambda^{2}} M^{-1} \mathbf{1},
$$

where $\alpha=\mathbf{1}^{\top} M^{-1} \mathbf{1}, \lambda=\mu^{\top} M^{-1} \mathbf{1}$ and $\theta=\mu^{\top} M^{-1} \mu$.

This model depends strictly on the assumptions that the assets returns follow normal distribution and investor has quadratic utility function. However, these two conditions are not satisfied. Many researchers have showed that the assets returns distribution are asymmetric and exhibit skewness, see Tobin (1958), Arditti (1971), Chunhachinda et al (1997) and Prakash et al (2003). These authors have proposed a DownSide Risk (DSR) measures such as Semivariance (SV) and conditional value at risk (CVaR). These DSR measures are consistent with investor's perception towards risk as they focus on return dispersions below specified target return $B$. Below, we focus only on the Semivariance risk measure which is often considered as a more plausible risk one than the variance. The associated optimization program is the following :

$$
\min _{\omega} \omega^{\top} M_{S R} \omega \text { subject to } \omega^{\top} \mu=E^{*}, \omega^{\top} \mathbf{1}=1,
$$


where $M_{S R}$ is the matrix with coefficients

$$
\Sigma_{i j B}=\frac{1}{T} \sum_{t=1}^{V}\left(r_{i t}-B\right)\left(r_{j t}-B\right)
$$

such that $V$ is the period in which the portfolio underperforms the target return $B$.

The major obstacle to get the solution of this problem is that the semicovariance matrix is endogenous (see Estrada 2004, 2008); that is, a change in weights affects the periods in which the portfolio underperforms the target rate of return, which in turn affects the elements of the Semivariance matrix.

Many authors propose different methods to estimate the elements of $M_{S R}$ in order to resolve problem defined in equation (4). Among them, Hogan and Warren (1974) propose to use the Frank-Wolf algorithm but the main disadvantage of this algorithm is its slow convergence rate. Moreover, during early iteration, this algorithm tends to decrease the objective function. Ang (1975) proposes to linearise the Semivariance so that the optimization problem can be solved using linear programming. However, this method ignores the inter-correlations between securities. Harlow (1991) also considers problem (4) and generates Mean-Semivariance efficient frontier, where he compares to the Mean-Variance efficient frontier. Mamoghli and Daboussi (2010) improve Harlow approach. Their model permits to surmount the problem of inequality of the Semicovariance measures which occur in the Mean-Semivariance model of Harlow. Markowitz et al (1993) transform the MeanSemivariance problem into a quadratic problem by adding fictitious securities. Estrada (2008) proposes a simple and accurate heuristic approach that yields a symmetric and exogenous Semicovariance matrix, which enables the determination of Mean-Semivariance optimal portfolios by using the well-known closed-form solutions of Mean-Variance problems. Athayde $(2001,2003)$ proposes an iterative algorithm and uses it to construct a Mean-DownSide Risk portfolio frontier. Ben Salah et al (2015a, 2015b) improve Athayde's algorithm by introducing nonparametric estimation of the returns in order to better smooth the efficient frontier.

A knowledgeable investor should have an overview on market development. For a fixed amount to invest now, he has to predict, day by day (or month by month, ...), the optimal return that his investment could bring to him.

In this paper, we will develop a rule of decision to optimize his portfolio by reducing the risk calculated by the classical variance. A new approach, called conditional Markowitz optimization method, will be used to determine an optimal portfolio. This portfolio is obtained by minimizing the so-called conditional risk. This risk may in turn be broken down into two versions : Mean-Variance and Mean-Semivariance approaches. The main idea is to anticipate the values of the assets returns on the date $T+1$ (knowing the past) and incorporate this information in the optimization model. 
The rest of the article is organized as follows. Section 2 introduces the nonparametric conditional risk. We start by given a general background on nonparametric regression and prediction, as well as conditional variance and covariance definitions. The conditional Mean-Variance and the conditional Mean-Semivariance models are exhibited in this Section. Numerical studies based on Chinese and American Market dataset are presented in Section 3. The last Section is devoted to conclusion and further development.

\section{Nonparametric Conditional Risk}

Here, we start by giving some general concepts and results concerning nonparametric regression and prediction. Then, we define the Conditional Mean-Variance and MeanSemivariance models and we exhibit the corresponding algorithms to get the optimal portfolio.

\subsection{Nonparametric Regression Model}

In the following subsection, we outline the mechanics of kernel regression estimation.

In what follows, we let $\left\{Y_{i}, X_{i}\right\}_{i=1}^{N}$ denote sample pairs of response and explanatory variables, where $Y_{i}$ is explanatory variables, $X_{i}$ is of dimension $m$, and $T$ denotes the sample size. The best prediction of $Y$ based on $X=x$ is the conditional expectation $E(Y \mid X=x)=g(x)$. The goal is to estimate the unknown mean response $g(x)$. In general, the function $g(x)$, is not necessarily linear and the conditional variance in not necessarily homoscedastic. However, we can always express the data in the form

$$
Y_{t}=g(x)+\sigma(x) \epsilon_{t},
$$

where $\sigma^{2}(x)=\operatorname{Var}(Y \mid X=x)$. Here $\epsilon_{t}$ has conditional zero mean and unit variance. The Nadaraya-Watson estimator for $g(x)$ is then :

$$
\hat{g}(x)=\frac{\sum_{t=1}^{N} Y_{t} \mathcal{K}\left(\frac{X_{t}-x}{h}\right)}{\sum_{t=1}^{N} \mathcal{K}\left(\frac{X_{t}-x}{h}\right)},
$$

where $\mathcal{K}$ is a multivariate kernel function with $m$ arguments and where $h$ is a sequence of positive numbers tending to 0 as $N$ tends ton infinity.

To simplify the writing and the understanding of previous predictors, we choose the following multiplicative expression for the multidimensional kernel $\mathcal{K}$ :

$$
\mathcal{K}(u)=K\left(u_{1}\right) \times \cdots \times K\left(u_{m}\right)
$$


where $K$ is an univariate kernel (density) function. Then (6), for $x=\left(x_{1}, \ldots, x_{m}\right)$, should be writing as follows :

$$
\hat{g}(x)=\frac{\sum_{t=1}^{N} Y_{t} K\left(\frac{X_{1 t}-x_{1}}{h}\right) \times \cdots \times K\left(\frac{X_{m t}-x_{m}}{h}\right)}{\sum_{t=1}^{N} K\left(\frac{X_{1 t}-x_{1}}{h}\right) \times \cdots \times K\left(\frac{X_{m t}-x_{m}}{h}\right)} .
$$

Nonparametric smoothing techniques can be applied beyond the estimation of the autoregression function. Consider a $m$-multivariate stationary time series $\left\{\left(r_{1 t}, \ldots, r_{m t}\right)\right.$, $t=1, \ldots, T\}$. We consider the processes $\left(X_{t}, Y_{t}\right)$ defined as follows

$$
X_{t}=\left(r_{1 t}, \ldots, r_{m t}\right) \quad Y_{t}=r_{j(t+1)},
$$

and we are interested in predicting the return of a given asset $j$ on time $T+1$. This problem is equivalent to the estimation of the regression function presented above

$$
g\left(r_{1 T}, \ldots, r_{m T}\right)=\mathbb{E}\left(Y_{T} \mid X_{T}=x_{T}\right)=\mathbb{E}\left(r_{j(T+1)} \mid R_{1 T}=r_{1 T}, \ldots, R_{m T}=r_{m T}\right) .
$$

Let $N=T-1$. Using the kernel method, we get easily the following estimators for conditional expectation, variance and covariance :

- Conditional expectation :

$$
\bar{r}_{c, j}=\frac{\sum_{t=1}^{N} r_{j(t+1)} K\left(\frac{r_{1 t}-r_{1 T}}{h}\right) \times \cdots \times K\left(\frac{r_{m t}-r_{m T}}{h}\right)}{\sum_{t=1}^{N} K\left(\frac{r_{1 t}-r_{1 T}}{h}\right) \times \cdots \times K\left(\frac{r_{m t}-r_{m T}}{h}\right)},
$$

- Conditional variance :

$$
\hat{\sigma}_{c, j}^{2}\left(r_{1 T}, \ldots, r_{m T}\right)=\frac{\sum_{t=1}^{N}\left(r_{j(t+1)}-\bar{r}_{c, j}\right)^{2} K\left(\frac{r_{1 t}-r_{1 T}}{h}\right) \times \cdots \times K\left(\frac{r_{m t}-r_{m T}}{h}\right)}{\sum_{t=1}^{N} K\left(\frac{r_{1 t}-r_{1 T}}{h}\right) \times \cdots \times K\left(\frac{r_{m t}-r_{m T}}{h}\right)},
$$

- Conditional covariance :

$$
\hat{\sigma}_{c, i j}\left(r_{1 T}, \ldots, r_{m T}\right)=\frac{\sum_{t=1}^{N}\left(r_{i(t+1)}-\bar{r}_{c, i}\right)\left(r_{j(t+1)}-\bar{r}_{c, j}\right) K\left(\frac{r_{1 t}-r_{1 T}}{h}\right) \times \cdots \times K\left(\frac{r_{m t}-r_{m T}}{h}\right)}{\sum_{t=1}^{N} K\left(\frac{r_{1 t}-r_{1 T}}{h}\right) \times \cdots \times K\left(\frac{r_{m t}-r_{m T}}{h}\right)},
$$

\section{Comments}

1. To estimate conditional expectation, variance and covariance, we supposed that the future depends on the immediate past. This hypothesis, considered as 1-Markovian condition, is natural because the future returns are strongly correlated to the recent past. 
2. In Statistics literature, Many other multivariate kernels are proposed like the Spherical/radialsymmetric kernel or the multivariate Epanechnikov (spherical) one.

3. Nonparametric methods are typically indexed by a bandwidth or tuning parameter which controls the degree of complexity. The choice of bandwidth is often critical to implementation : under- or over-smoothing can substantially reduce precision. The standard approach to the bandwidth problem is to choose a bandwidth that minimizes some measure of global risk for the entire regression function, usually Mean Integrated Squared Error (MISE), i.e. the expected squared error integrated over the entire curve. The optimal bandwidth is then estimated either using plug-in estimators of the minimizer of the asymptotic approximation to MISE or using an unbiased databased estimator of the MISE . This is the cross-validation method. This method was analyzed in Sarda (1993). It is recommended by Altman and Leger (1995) when large samples are available. As to the estimation of the conditional covariance matrix, one may use different bandwidths for different elements of this matrix. However, the resulting estimate with different bandwidths cannot be guaranteed to be positive definite (Li et al, 2007). In practice, the positive definiteness is a desirable property. Thus, we suggest using the same bandwidth for all elements.

\subsection{Conditional Mean-Variance Model}

Our goal here is to constitute an optimal portfolio using conditional criterion. In our opinion, it is natural to use conditional informations to provide a timely and effective solution.

We suppose that they are, as in the previous section, $m$ assets to be used for constructing a well diversified portfolio. Optimizing asset allocation is simply defined as the process of mixing asset weights of a portfolio within the constraints of an investor's capital resources to yield the most favourable risk-return trade-off. The risk here is defined by conditional variance the portfolio return.

Let $\omega=\left(\omega_{1}, \ldots, \omega_{m}\right)^{\top}$ be the portfolio weight vector and $r_{p t}=\omega_{1} r_{1 t}+\omega_{2} r_{2 t}+\ldots+$

$\omega_{m} r_{m t}, t=1 \ldots, T, T$-realisations of the the portfolio return $R_{p}$. Using conditional meanvariance criterion, the optimization model is

$$
\min _{\omega} \omega^{\top} M_{c} \omega, \text { subject to } \omega^{\top} \mu_{c}=E^{*}, \omega^{\top} \mathbf{1}=1,
$$

where $\mu_{c}=\left(\bar{r}_{c, 1}, \ldots, \bar{r}_{c, m}\right)^{\top}, E^{*}$ is a given expected return and $M_{c}$ is the conditional covariance matrix whose elements are $\hat{\sigma}_{c, i j}\left(r_{1 T}, \ldots, r_{m T}\right)$.

Using Lagrangian method, an explicite solution of (13) is given by :

$$
\omega_{c, *}=\frac{\alpha_{c} E^{*}-\lambda_{c}}{\alpha_{c} \theta_{c}-\lambda_{c}^{2}} M_{c}^{-1} \mu_{c}+\frac{\theta_{c}-\lambda_{c} E^{*}}{\alpha_{c} \theta_{c}-\lambda_{c}^{2}} M_{c}^{-1} \mathbf{1}
$$

where $\alpha_{c}=\mathbf{1}^{\top} M_{c}^{-1} \mathbf{1}, \lambda_{c}=\mu_{c}^{\top} M_{c}^{-1} \mathbf{1}$ and $\theta_{c}=\mu_{c}^{\top} M_{c}^{-1} \mu_{c}$. 
Then the conditional risk, $C R$, is given by

$$
C R=\left(\omega_{c, *}^{\top} M_{c} \omega_{c, *}\right) .
$$

\subsection{Conditional Mean-Semivariance Model}

Let us recall that the main criticism to variance, used by Markowitz (1952) as measure of risk is, in essence, that it gives the same importance and the same weight to gains and losses, also the use of variance suppose that returns are normally distributed . That is why Markowitz (1959) argues for another more plausible measure of risk that he calls the Semivariance wich takes into consideration the assymetry and the risk perception of investors. For motivations, details and theortical result on this method, see for example Vasant et al. (2014).

Let $B$ the Benchmark (a threshold which captured the risk perspectives from investors to investors). It is a target return which can be equal to 0 . The conditional meanSemivariance model is the following :

$$
\min _{\omega} \omega^{\top} M_{\{c, S R\}} \omega \text { subject to } \omega^{\top} \mu_{c}=E^{*}, \omega^{\top} \mathbf{1}=1,
$$

where $\left.M_{\{c, S R}\right\}$ is the matrix with coefficients

$$
\hat{\sigma}_{i j B}=\frac{\sum_{t=1}^{V}\left(r_{i t}-B\right)\left(r_{j t}-B\right) K\left(\frac{r_{1 t}-r_{1 T}}{h}\right) \times \cdots \times K\left(\frac{r_{m t}-r_{m T}}{h}\right)}{\sum_{t=1}^{V} K\left(\frac{r_{1 t}-r_{1 T}}{h}\right) \times \cdots \times K\left(\frac{r_{m t}-r_{m T}}{h}\right)},
$$

such that $V$ is the period in which the portfolio underperforms the target return $B$.

Remark. The coefficients $\hat{\sigma}_{i j B}$ are computed differently of those of (12). To do it in the same way, we should reindex the observations (such that portfolio underperforms the target return $B$ ) in order to get a new time series process and then apply (12). This modification is not very helpful given the abundance of data.

Resolving this problem is not easier. Based on Athayde algorithm (2001), we develop, in the following, an iterative algorithm that could resolve the optimization problem without enormous difficulties. The principle is the use of Lagrangian method at each step.

- Step 0 :

- Start with $\omega_{c, 0}=\left(\omega_{c, 0}^{1}, \ldots, \omega_{c, 0}^{m}\right)$,

- compute $r_{p t}^{0}=\omega_{c, 0}^{1} r_{1 t}+\cdots+\omega_{c, 0}^{m} r_{m t}, t=1, \ldots, T$,

- select the set $S_{0}$ of time indices portfolio return observations in which this portfolio $\omega_{c, 0}$ had negative deviations i.e. $r_{p t}^{0}-B \leq 0$.

- Construct the following positive $(m \times m)$ semi-definite matrix $M_{\{c, S R, 0\}}$ : 


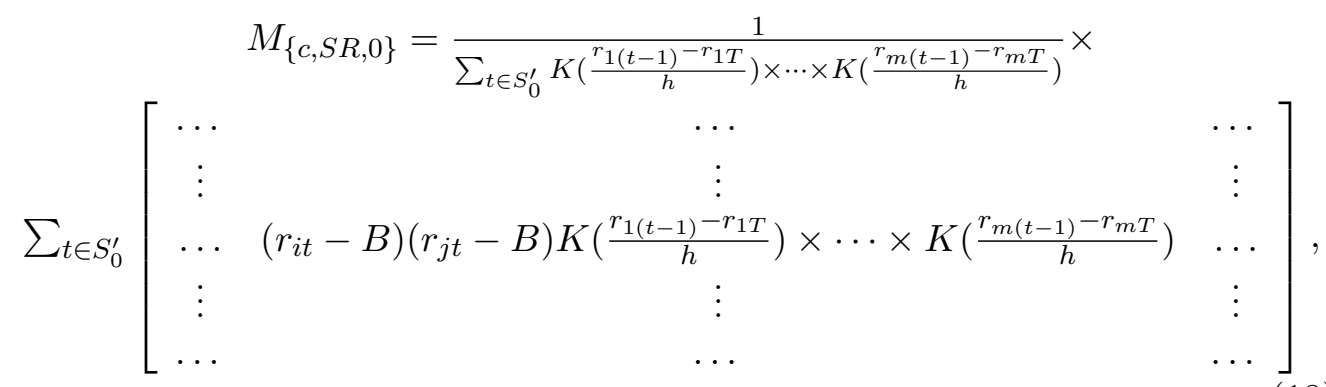

where $S_{0}^{\prime}=S_{0}$ or $S_{0}=S_{0} \backslash\{1\}\left(\right.$ if $\left.1 \in S_{0}\right)$.

- Step 1 : find the portfolio $\omega_{c, 1}$ that solves the following problem :

$$
\min _{\omega} \omega^{\top} M_{\{c, S R, 0\}} \omega \text { subject to } \omega^{\top} \mu_{c}=E^{*}, \omega^{\top} \mathbf{1}=1 \text {. }
$$

Using Lagrangian Method, the solution to the problem (19) will be given by :

$$
\omega_{c, 1}=\frac{\alpha_{c, 1} E^{*}-\lambda_{c, 1}}{\alpha_{c, 1} \theta_{c, 1}-\lambda_{c, 1}^{2}}\left(M_{\{c, S R, 0\}}\right)^{-1} \mu_{c}+\frac{\theta_{c, 1}-\lambda_{c, 1} E^{*}}{\alpha_{c} \theta_{c, 1}-\lambda_{c, 1}^{2}}\left(M_{\{c, S R, 0\}}\right)^{-1} \mathbf{1},
$$

where $\alpha_{c, 1}=\mathbf{1}^{\top}\left(M_{\{c, S R, 0\}}\right)^{-1} \mathbf{1}, \lambda_{c, 1}=\mu_{c, 1}^{\top}\left(M_{\{c, S R, 0\}}\right)^{-1} \mathbf{1}$ and $\theta_{c, 1}=\mu_{c}^{\top}\left(M_{\{c, S R, 0\}}\right)^{-1} \mu_{c}$.

\section{- Step 2 :}

- compute $r_{p t}^{1}=\omega_{c, 1}^{1} r_{1 t}+\cdots+\omega_{c, 1}^{m} r_{m t}, t=1, \ldots, T$,

- select the set $S_{1}$ of index observations in which this portfolio $\omega_{c, 1}$ had negative deviations i.e. $r_{p t}^{1}-B \leq 0$,

- construct the following positive $(m \times m)$ semi-definite matrix $M_{\{c, S R, 1\}}$ :

$$
\begin{gathered}
M_{\{c, S R, 1\}}=\frac{1}{\sum_{t \in S_{1}^{\prime}} K\left(\frac{r_{1(t-1)}-r_{1 T}}{h}\right) \times \cdots \times K\left(\frac{r_{m(t-1)}-r_{m T}}{h}\right)} \times \\
\sum_{t \in S_{1}}\left[\begin{array}{ccc}
\cdots & \ldots & \cdots \\
\vdots & \vdots & \vdots \\
\ldots & \left(r_{i t}-B\right)\left(r_{j t}-B\right) K\left(\frac{r_{1(t-1)}-r_{1 T}}{h}\right) \times \cdots \times K\left(\frac{r_{m(t-1)}-r_{m T}}{h}\right) & \ldots \\
\cdots & \vdots & \vdots \\
\cdots & \cdots & \cdots
\end{array}\right],
\end{gathered}
$$

where $S_{1}^{\prime}=S_{1}$ or $S_{1}^{\prime}=S_{1} \backslash\{1\}$ (if $1 \in S_{1}$ ).

- find the portfolio $\omega_{c, 2}$ that solves the following problem :

$$
\min _{\omega} \omega^{\top} M_{\{c, S R, 1\}} \omega \text { subject to } \omega^{\top} \mu_{c}=E^{*}, \omega^{\top} \mathbf{1}=1 .
$$

Using Lagrangian Method, the solution to the problem (22) will be given by :

$$
\omega_{c, 2}=\frac{\alpha_{c, 2} E^{*}-\lambda_{c, 2}}{\alpha_{c, 2} \theta_{c, 2}-\lambda_{c, 2}^{2}}\left(M_{\{c, S R, 1\}}\right)^{-1} \mu_{c}+\frac{\theta_{c, 2}-\lambda_{c, 2} E^{*}}{\alpha_{c} \theta_{c, 2}-\lambda_{c, 2}^{2}}\left(M_{\{c, S R, 1\}}\right)^{-1} \mathbf{1} .
$$


- Step 3 : iterate the previous process to construct a sequence of matrices $M_{\{c, S R, l\}}$ until getting the first matrix $M_{\{c, S R, F\}}$ satisfying the criterion $M_{\{c, S R, F\}}=M_{\{c, S R, F+1\}}$. The Optimal portfolio will be given by :

$$
\omega_{c, F+1}=\frac{\alpha_{c, F} E^{*}-\lambda_{c, F}}{\alpha_{c, F} \theta_{c, F}-\lambda_{c, F}^{2}}\left(M_{\{c, S R, F\}}\right)^{-1} \mu_{c}+\frac{\theta_{c, F}-\lambda_{c, F} E^{*}}{\alpha_{c} \theta_{c, F}-\lambda_{c, F}^{2}}\left(M_{\{c, S R, F\}}\right)^{-1} \mathbf{1} .
$$

and the Conditional Semi Risk value, $C S R$, will be

$$
C S R=\omega_{c, F+1}^{\top} M_{\{c, S R, F\}} \omega_{c, F+1}
$$

\section{Remarks.}

1. There is a finite number of iterations to get the optimal solution.

2. In the prediction step (to get the an unobservable values of the returns), we treated separately the evolution of each asset. It is possible to make multivariate (or vectorial) prediction and get jointly the an unobservable values for all the assets.

3. Short selling is allowed in this model, i.e. the optimal portfolio can have a negative weight for some assets. To forbid short selling, the additional constraint $\omega_{j} \geq 0$ for $j=1, \ldots, m$. is necessary.

\section{Empirical Analysis}

In this section, the performance of the proposed methods are investigated. Classical Markowitz and DownSide methods are compared to the proposed method. It is supposed that there is no transaction costs, no taxes and the Benchmark $B=0$.

\subsection{Data}

A dataset, drawn from Reuters, was used for this analysis. The original Data consist on daily stock returns belonged to two markets :

- the Chinese market (emerging market) with 16 assets. They are 897 daily observations (returns) for each asset from November, the 14th, 2011, to July, 8th, 2015,

- the American market (developed market) with 19 assets. They are 788 daily observations (returns) for each asset from June, 18th, 2012 to July, 8th, 2015.

To compare the efficiency and the performance of the proposed methods, we use the daily values of

- the Hang Seng Index-HSI that aims to capture the leadership of the Hong Kong exchange, and covers approximately $65 \%$ of its total market capitalization,

- the S\&P 500 Index that tracks 500 large U.S. companies across a wide span of industries and sectors. The stocks in the S\&P 500 represent roughly $70 \%$ of all the stocks that are publicly traded. 
The assets returns are calculated from stock prices observed on Thomson Reuters Platform as follows :

$$
r_{t}=\frac{p_{t}-p_{t-1}}{p_{t-1}}
$$

with

- $p_{t}:$ Stock price at date $t$

$-p_{t-1}:$ Stock price at date $t-1$

The prices $p_{t}, t=1, \ldots, T$; are adjusted for dividends.

The historical statistics of the asset markets are summarised below.

\subsection{Historical statistics}

In this subsection, historical statistics are proposed. The goal is to check the normality or not of the returns distribution in order to decide which risk measure is more appropriate to determine the optimal portfolio.

\subsubsection{Chinese Market}

Let us start by the Chinese Market. Over the past two decades, the Chinese economy and financial markets have undergone a remarkable transformation and seen significant growth. More specifically, the Chinese equity market has grown from a once very rudimentary and closed market to one of the largest equity markets in the world. Although most of the Chinese equity market still remains in the hands of controlling parties and domestic investors, authorities have made significant progress in opening the market to foreign capital and increasing the tradable float outstanding-meaning the Chinese equity market has the potential to become a top dominant force within global portfolios. 
TABLE 1 - Chinese historical analysis

\begin{tabular}{llrrrrrr}
\hline & Abbreviation & Min & Mean & Sd & Skewness & Kurtosis & Max \\
\hline HangSeng & HSI & -5.84 & 0.03 & 1.01 & -0.22 & 1.86 & 3.80 \\
Agricultural.Bk & A.Bk & -9.90 & 0.06 & 1.61 & 0.70 & 9.93 & 10.12 \\
Bank.of.China & B.Ch & -10.98 & 0.09 & 1.78 & 0.69 & 11.31 & 10.14 \\
Ind.And.Com.Bank & IACB & -9.90 & 0.04 & 1.48 & 0.06 & 9.18 & 9.04 \\
Petrochina & Pet & -9.21 & 0.04 & 1.68 & 0.87 & 12.09 & 10.04 \\
China.life.insurance & Ch.L.I. & -10.01 & 0.10 & 2.40 & 0.71 & 4.02 & 10.04 \\
China.petroleum & Ch.P. & -10.04 & 0.04 & 1.88 & 0.28 & 6.85 & 10.04 \\
Bank.of.Com. & Bk.C. & -10.00 & 0.08 & 1.97 & 0.74 & 8.88 & 10.10 \\
Citic.securit & Ci.Se & -7.14 & 0.00 & 1.81 & 1.14 & 6.81 & 12.95 \\
China.telecom & Ch.T. & -4.85 & 0.01 & 1.81 & 0.48 & 1.49 & 9.28 \\
China.pacific & Ch.P. & -9.98 & 0.08 & 2.38 & 0.55 & 3.51 & 13.50 \\
Chinarailway & Ch.R. & -10.14 & 0.19 & 2.92 & 2.65 & 20.68 & 26.59 \\
Huaneng & Hua & -10.35 & 0.11 & 2.35 & 0.21 & 5.90 & 14.83 \\
Greatwall & Gre & -10.00 & 0.18 & 2.63 & 0.19 & 1.38 & 10.63 \\
Dong.feng & Do.F. & -6.96 & -0.01 & 2.19 & 0.26 & 0.55 & 8.13 \\
China.nat.buil & Ch.N.B & -8.96 & -0.03 & 2.16 & 0.45 & 2.39 & 11.12 \\
Tsingtao & Tsi & -10.04 & 0.01 & 1.79 & 0.27 & 6.86 & 12.79 \\
\hline
\end{tabular}

Taking all assets together, we observe that

- $-10.98 \leq$ Min $\leq-4.85$

- $3.80 \leq$ Max $\leq 26.59$

- $-0.03 \leq$ Mean $\leq 0.19$

- $1.01 \leq S D \leq 2.92$

- $0.55 \leq$ Kurtosis $\leq 20.68$

- $-0.22 \leq$ Skwenes $\leq 2.65$

It means that the behaviour of assets return is different from an asset to another. However, the returns distribution are more peaked than a Gaussian distribution (the Skewness $\neq 0$ ). This statement should favour the use of the DSR methods to optimize a portfolio. As a first time, we process the data as they are normally distributed. Our first goal is to compare the efficiency of classical Markowitz Mean-Variance method versus the proposed Conditional-Mean-Variance method. The second one is to compare the Mean-DownSide Risk method to the Conditional one.

To analyse the possible correlation between the different assets, It is helpfull to know the correlation matrix. This matrix is presented in Table (2) 
TABle 2 - China Correlation Matrix

\begin{tabular}{rrrrrrrrrrrrrrrrrr}
\multicolumn{11}{c}{ TABLE $2-$ China Correlation Matrix } \\
\hline \multicolumn{110}{c}{ A.Bk B.Ch IACB } & Pet C.L.I & \multicolumn{11}{c}{ Ch.P. Bk.C. Ci.Se Ch.T. Ch.P. Ch.R. Hua } & Gre Do.F. C.N.B & Tsi \\
\hline A.Bk & 1.00 & 0.82 & 0.80 & 0.57 & 0.49 & 0.59 & 0.76 & 0.04 & 0.04 & 0.25 & 0.18 & 0.01 & -0.03 & 0.01 & 0.03 & 0.09 \\
B.Ch & 0.82 & 1.00 & 0.76 & 0.55 & 0.49 & 0.57 & 0.74 & 0.03 & 0.00 & 0.23 & 0.23 & -0.00 & -0.04 & -0.01 & -0.02 & 0.05 \\
IACB & 0.80 & 0.76 & 1.00 & 0.60 & 0.45 & 0.60 & 0.72 & 0.02 & 0.05 & 0.20 & 0.16 & -0.01 & -0.03 & 0.01 & 0.05 & 0.09 \\
Pet & 0.57 & 0.55 & 0.60 & 1.00 & 0.54 & 0.74 & 0.52 & 0.02 & 0.06 & 0.23 & 0.18 & 0.00 & 0.00 & 0.02 & 0.06 & 0.09 \\
C.L.I & 0.49 & 0.49 & 0.45 & 0.54 & 1.00 & 0.45 & 0.49 & -0.01 & 0.04 & 0.23 & 0.23 & 0.03 & -0.02 & 0.01 & 0.03 & 0.09 \\
Ch.P. & 0.59 & 0.57 & 0.60 & 0.74 & 0.45 & 1.00 & 0.57 & 0.09 & 0.04 & 0.19 & 0.18 & 0.02 & -0.00 & 0.06 & 0.11 & 0.06 \\
Bk.C. & 0.76 & 0.74 & 0.72 & 0.52 & 0.49 & 0.57 & 1.00 & 0.03 & 0.01 & 0.26 & 0.19 & -0.01 & -0.03 & 0.00 & -0.00 & 0.11 \\
Ci.Se & 0.04 & 0.03 & 0.02 & 0.02 & -0.01 & 0.09 & 0.03 & 1.00 & 0.10 & 0.05 & 0.03 & -0.01 & -0.01 & 0.12 & 0.13 & 0.03 \\
Ch.T. & 0.04 & 0.00 & 0.05 & 0.06 & 0.04 & 0.04 & 0.01 & 0.10 & 1.00 & 0.01 & 0.05 & -0.01 & -0.02 & 0.12 & 0.40 & -0.03 \\
Ch.P. & 0.25 & 0.23 & 0.20 & 0.23 & 0.23 & 0.19 & 0.26 & 0.05 & 0.01 & 1.00 & 0.15 & -0.03 & 0.01 & 0.00 & 0.01 & 0.13 \\
Ch.R. & 0.18 & 0.23 & 0.16 & 0.18 & 0.23 & 0.18 & 0.19 & 0.03 & 0.05 & 0.15 & 1.00 & 0.05 & 0.00 & 0.01 & 0.01 & 0.16 \\
Hua & 0.01 & -0.00 & -0.01 & 0.00 & 0.03 & 0.02 & -0.01 & -0.01 & -0.01 & -0.03 & 0.05 & 1.00 & 0.00 & 0.01 & 0.06 & 0.08 \\
Gre & -0.03 & -0.04 & -0.03 & 0.00 & -0.02 & -0.00 & -0.03 & -0.01 & -0.02 & 0.01 & 0.00 & 0.00 & 1.00 & 0.02 & 0.03 & 0.03 \\
Do.F. & 0.01 & -0.01 & 0.01 & 0.02 & 0.01 & 0.06 & 0.00 & 0.12 & 0.12 & 0.00 & 0.01 & 0.01 & 0.02 & 1.00 & 0.12 & 0.00 \\
C.N.B & 0.03 & -0.02 & 0.05 & 0.06 & 0.03 & 0.11 & -0.00 & 0.13 & 0.40 & 0.01 & 0.01 & 0.06 & 0.03 & 0.12 & 1.00 & -0.01 \\
Tsi & 0.09 & 0.05 & 0.09 & 0.09 & 0.09 & 0.06 & 0.11 & 0.03 & -0.03 & 0.13 & 0.16 & 0.08 & 0.03 & 0.00 & -0.01 & 1.00 \\
\hline
\end{tabular}

It appears that the returns are not correlated between each other similarly. For example, Agricultural.Bk is strongly correlated to Bank.of.China and Ind.And.Com.Bank assets, and very weakly correlated to Dong.feng and China.nat.buil assets. More generally, the correlation between two stocks is larger when they are from banking sector than when they belong to different industries. The use of Principal Component Analysis (PCA) could reduce the number of assets to constitute the optimal portfolio. This step is omitted in this paper.

\section{Remark.}

We have excluded, in this matrix, the correlation between the HangSeng Index and the other assets because we will use it as a benchmark to compare its return to the optimal portfolio one.

\subsubsection{The Americain Market : S\&P 500}

The S\&P 500 is designed to be a leading indicator of U.S. equities and is meant to reflect the risk/return characteristics of the large cap universe. Companies included in the index are selected by the S\&P Index Committee, a team of analysts and economists at Standard \& Poor's. The S\&P 500 is a market value weighted index, each stock's weight is proportionate to its market value.

Similar historical statistics are done for this Market. In the beginning, we exhibit and comment the descriptive statistics, then we exhibit and comment the correlation matrix. 
TABLE 3 - USA Historical Statistics

\begin{tabular}{lccccccc}
\hline \multicolumn{1}{c}{} & Abbreviation & Min & Mean & SD & Skewness & Kurtosis & Max \\
\hline SP500 & S\&P & -2.50 & 0.06 & 0.73 & -0.15 & 0.99 & 2.54 \\
Apple & App & -12.37 & 0.07 & 1.62 & -0.54 & 6.81 & 8.20 \\
Google & Goo & -8.09 & 0.09 & 1.34 & 1.28 & 16.27 & 13.86 \\
Microsoft & Mft & -11.40 & 0.06 & 1.44 & -0.23 & 11.57 & 10.40 \\
Exxon & Exx & -4.18 & 0.00 & 0.94 & -0.11 & 1.57 & 3.22 \\
Berkshire & Ber & -0.90 & 0.03 & 0.42 & -1.3 & 14.29 & 9.34 \\
Wellsfargo & Wel & -4.98 & 0.08 & 1.04 & 0.07 & 1.92 & 4.37 \\
Johnson & Joh & -2.88 & 0.05 & 0.84 & -0.22 & 0.85 & 2.57 \\
General.Electric & Gel & -5.35 & 0.04 & 1.07 & 0.33 & 4.74 & 7.34 \\
JP.Morgan & JPM & -5.49 & 0.08 & 1.24 & -0.07 & 1.49 & 5.48 \\
Facebook & Fbk & -12.12 & 0.17 & 2.73 & 2.18 & 21.88 & 29.56 \\
Wallmart & Wal & -4.36 & 0.01 & 0.89 & -0.23 & 2.81 & 4.72 \\
Procter.and.Gramble & PaG & -6.44 & 0.04 & 0.88 & -0.20 & 5.68 & 4.01 \\
Pfizer & Pfi & -4.41 & 0.06 & 0.97 & 0.00 & 1.58 & 4.23 \\
Amazon & Ama & -11.10 & 0.10 & 1.87 & 0.54 & 11.98 & 14.11 \\
Walt.Disney & WDi & -8.21 & 0.12 & 1.22 & 0.42 & 10.48 & 9.89 \\
Coca.Cola & CCO & -5.96 & 0.01 & 0.92 & -0.08 & 4.65 & 5.44 \\
Gilead.sciences & GiS & -14.34 & 0.21 & 1.89 & -0.06 & 8.52 & 13.72 \\
Visa & Vis & -7.14 & 0.11 & 1.27 & 0.59 & 7.33 & 10.29 \\
Citi.group & CGr & -6.22 & 0.10 & 1.48 & 0.16 & 1.50 & 6.42 \\
\hline
\end{tabular}

Taking all assets together, we observe that

- $-14.34 \leq$ Min $\leq-0.90$

- $2.54 \leq$ Max $\leq 29.56$

- $0.0 \leq$ Mean $\leq 0.21$

- $0.73 \leq S D \leq 2.73$

- $0.85 \leq$ Kurtosis $\leq 21.88$

- $-0.54 \leq$ Skwenes $\leq 2.18$

The behaviour of assets return is different from an asset to another. However, Most of distributions are more peaked than a Gaussian distribution (the Skewness $\neq 0$ ). The SP 500 index could be considered as is normally distributed (Skewness=-0.15, Kurtosis=0.99).

Table (4) is devoted to the Correlation Matrix : 
TABLE 4 - USA Correlation Matrix

\begin{tabular}{|c|c|c|c|c|c|c|c|c|c|c|c|c|c|c|c|}
\hline App & oo Mtt & & $x_{1}$ & Joh & & $P_{M}$ & $\mathrm{Fb}$ & & $\mathrm{PaG}$ & & $a$ & WV & & Gis & \\
\hline App 1.00 & & & & & & & & & & & & & & 16 & \\
\hline & & & & & & & & & & & & & & & \\
\hline & & & & & & & & & & & & & & & \\
\hline & & & & & & & & & & & & & 38 & 29 & \\
\hline & & & & & & & & & & & 01 & & 00 & 09 & .02 \\
\hline & & & & & & & & & & & & & 38 & 34 & .66 \\
\hline & & & & & & & & & & 01 & 29 & .43 & 0.44 & 0 & .43 \\
\hline & & & & & & & & & & 41 & & & & .02 & .01 \\
\hline & & & & & & & & & & 02 & & 44 & 34 & 29 & 0.77 \\
\hline & & & & & & & & & & 04 & 22 & 21 & 07 & & 0.17 \\
\hline & & & & & & & & & & 02 & 20 & 41 & 38 & 20 & .31 \\
\hline & & & & & & & 06 & & & 05 & 17 & & 49 & & .32 \\
\hline & & & & & & & & & & 00 & 04 & & 03 & 06 & .01 \\
\hline & & & & & & & & & & & & & & 27 & .33 \\
\hline & & & & & & & & & & 01 & & & & 26 & .42 \\
\hline & & & & & & & & & & & & & & & .34 \\
\hline & & & & & & & & & & & & & & & .32 \\
\hline & & & & & & & & & & & & & & .07 & 0.06 \\
\hline $\mathrm{CGr}$ & 390.33 & tis & 0.020 .66 & 0.10 & 0.01 & & 0.18 & & 0.32 & 0.01 & 0.33 & 0.42 & 0.34 & 0.32 & 0.061 .00 \\
\hline
\end{tabular}

The correlation between two stocks is larger when they are from similar sectors, for example Facebook, Google, Amazon and JP Morgan, City Group and Johnson are positively correlated. There is no significant negative correlation. All the correlation are low or medium.

\subsection{Portfolio Optimisation}

Using the data of the two markets, we will use the Conditional Mean-Variance and the Conditional Mean-Semivariance models to get optimal portfolios that we can invest in each market. The idea is to anticipate the future knowing the past. In the classical methods, Mean, Variance and Semivariance take no account the forthcoming data.

Our methodology consists on dividing the data into two samples : one for making the optimization (optimization sample) and the other for testing the efficiency of the methods (test sample). The optimization sample is used to determine the optimal weights for each method (classical Mean-Variance, Conditional Mean-Variance, Classical Mean-Semivariance and Conditional Mean-Semivariance). These weights are used for computing the optimal portfolio returns for each method.

In order to measure the performance of the proposed methods we use the sample test. The optimal portfolio returns are compared to the naive one and they are also used to assess performance against the HangSeng index and S\&P 500 one.

The following parameters and considerations will be used throughout this section :

- Optimal portfolio is determined for one period,

- for the expected return $E^{*}$, many values are tested. We have decided to present only 
results with $E^{*}=0.075 \%$

- the Benchmark $B=0$,

- the kernel $\mathcal{K}$ is the multivariate Gaussian density, $\mathcal{K}(x)=\frac{1}{(2 \pi)^{n / 2}} \exp -\left(\frac{x_{1}^{2}+\cdots+x_{m}^{2}}{2}\right)$,

- the bandwidths $h$ are chosen by cross validation method and depend on each asset return observations (see previous chapters). This choice is motivated by its popularity in nonparametric literature (see Arlot and Celisse (2010)).

- there is no transaction cost,

- short selling is allowed.

To test our methods, we used the following procedure :

- to determine the optimal portfolio, we use all the data collected until May, 31th, 2015 ,

- returns collected from June, the 1st, 2015 until July, the 7th, 2015 are used to compare naive portfolio return (obtained using $\omega_{i}=\frac{1}{m}$ ) to those obtained by the other methods.

For the Chinese Market, results are summarized in Table (5). Table (7) is devoted to the American Market. From now on, the following abreviation will be used :

- M-V= Classical Mean-Varince Model

- C.M-V = Conditional Mean-Varince Model

- M-DSR = Classical Mean-Semivarince Model

- C.M-DSR $=$ Conditional Mean-Semivarince Model 


\begin{tabular}{cccccc}
\multicolumn{5}{c}{ TABLE } & - Chinese Portfolio return $(\%)$ \\
\hline Time & M-V. & C.M-V & DSR & C.M-DSR Naive \\
2015-06-01 & 1.50 & 1.41 & 1.55 & 1.60 & 1.32 \\
$2015-06-02$ & 0.67 & 0.75 & 0.76 & 0.95 & 0.85 \\
$2015-06-03$ & 2.02 & 2.18 & 2.25 & 2.49 & 1.28 \\
$2015-06-04$ & -0.40 & -0.32 & -0.23 & -0.20 & -0.33 \\
$2015-06-05$ & -0.59 & -0.81 & -0.52 & -0.64 & -0.22 \\
$2015-06-08$ & 1.07 & 2.95 & 1.76 & 3.07 & 3.79 \\
$2015-06-09$ & -2.22 & -1.77 & -1.56 & -1.16 & -1.87 \\
$2015-06-10$ & -0.66 & -1.01 & -0.57 & -0.17 & -0.87 \\
$2015-06-11$ & 0.50 & 0.71 & 0.59 & 0.29 & -0.49 \\
$2015-06-12$ & 0.71 & 0.75 & 0.79 & 0.70 & -0.21 \\
$2015-06-15$ & -1.94 & -2.12 & -1.94 & -1.25 & -1.50 \\
$2015-06-16$ & -2.47 & -2.05 & -2.01 & -2.71 & -2.62 \\
$2015-06-17$ & 0.48 & 0.60 & 0.76 & 0.85 & 0.58 \\
$2015-06-18$ & -1.24 & -1.15 & -0.98 & -1.30 & -2.63 \\
$2015-06-19$ & -1.77 & -4.09 & -1.74 & -4.05 & -4.17 \\
$2015-06-22$ & 2.41 & 3.38 & 2.59 & 3.53 & 1.75 \\
$2015-06-23$ & 1.83 & 1.83 & 1.85 & 1.95 & 1.61 \\
$2015-06-24$ & -0.69 & -0.36 & -0.54 & -0.25 & -1.10 \\
$2015-06-25$ & -4.00 & -2.60 & -1.96 & -2.04 & -4.54 \\
$2015-06-26$ & 0.90 & 2.90 & 1.96 & 1.73 & 0.17 \\
$2015-06-29$ & 0.20 & 1.89 & 1.90 & 2.23 & 0.62 \\
$2015-06-30$ & -1.41 & -0.40 & -1.33 & -2.90 & -3.05 \\
$2015-07-02$ & 1.05 & 1.52 & 1.96 & 1.95 & 1.27 \\
$2015-07-03$ & -0.32 & -0.48 & -0.17 & -0.22 & -3.83 \\
$2015-07-06$ & 1.23 & 1.25 & 0.98 & 1.27 & 4.56 \\
$2015-07-07$ & -1.90 & -1.58 & -1.13 & -2.36 & -2.82
\end{tabular}

From Table (5) this table, we can extract the following comparative Table (6) which could inform us about the efficiency of each method compared to effective return obtained by a naive portfolio, computed day after day starting from June, the 1st, 2015. Below, the numerator of the fraction is the number of days on which methods perform better than the naive one.

TABLE 6 - Naive versus the other portfolio optimization methods in the Chinese Market Naive M-V. C.M-V M-DSR C.M-DSR Naive $26 / 26 \quad 16 / 26 \quad 20 / 26 \quad 21 / 26 \quad \mathbf{2 2} / \mathbf{2 6}$

In the same spirit and using the same conditions, the portfolio optimization processes, in the American Market, are summarized in Table (7) 


\begin{tabular}{|c|c|c|c|c|}
\hline Time & C.M.V & $\mathrm{DS}$ & C.D & Naive \\
\hline 2015-06-01 -0.12 & -0.11 & -0.10 & 0.12 & 0.41 \\
\hline 2015-06-02 -0.08 & -0.02 & -0.02 & -0.03 & -0.04 \\
\hline $015-06-03-0.03$ & 0.13 & 0.05 & 0.15 & 0.07 \\
\hline 2015-06-04 -0.77 & -0.60 & -0.57 & -0.51 & -0.80 \\
\hline 2015-06-05 -0.91 & -0.68 & -0.85 & -0.65 & -0.88 \\
\hline 2015-06-08 0.07 & -0.15 & 0.07 & 0.08 & -0.58 \\
\hline 2015-06-09 0.22 & 0.26 & 0.27 & 0.57 & 0.21 \\
\hline 2015-06-10 0.50 & 0.73 & 0.48 & 0.80 & 0.75 \\
\hline 2015-06-11 -0.20 & -0.19 & -0.24 & -0.15 & 0.13 \\
\hline 2015-06-12 -0.75 & -0.62 & -0.70 & -0.55 & -0.68 \\
\hline 2015-06-15-0.78 & -0.81 & -0.81 & -0.67 & -0.69 \\
\hline 2015-06-16 0.67 & 0.81 & 0.78 & 0.85 & 0.63 \\
\hline 2015-06-17 0.45 & 0.51 & 0.44 & 0.56 & 0.15 \\
\hline 2015-06-18 0.73 & 0.77 & 0.88 & 0.95 & 1.19 \\
\hline 2015-06-19-0.37 & -0.51 & -0.37 & -0.28 & -0.75 \\
\hline 2015-06-22 0.09 & 0.62 & 0.41 & 0.53 & 0.34 \\
\hline $2015-06-23-0.22$ & -0.06 & -0.22 & -0.04 & 0.44 \\
\hline $2015-06-24-0.38$ & -0.58 & -0.41 & -0.25 & -0.50 \\
\hline $2015-06-25-0.65$ & -0.17 & -0.67 & -0.08 & -0.49 \\
\hline 2015-06-26 0.07 & 0.10 & 0.11 & 0.22 & -0.03 \\
\hline 2015-06-29 -1.17 & -1.41 & -1.20 & -1.10 & -1.98 \\
\hline $2015-06-30-0.45$ & -0.45 & -0.43 & -0.21 & -0.55 \\
\hline 2015-07-01 0.81 & 1.28 & 0.86 & 1.48 & 0.73 \\
\hline 2015-07-02 0.13 & 0.11 & 0.21 & 0.14 & -0.07 \\
\hline $2015-07-06-0.35$ & -0.28 & -0.30 & -0.15 & -0.17 \\
\hline $2015-07-07 \quad 1.50$ & 1.22 & 1.48 & 1.55 & 1.47 \\
\hline
\end{tabular}

To compare all the methods, we use, as in Chinese Market, the naive portfolio as the reference portfolio and the following comparative table (Table 8)

TABLE 8 - Naive versus the other portfolio optimization methods in the American Market Naive M-V. C.M-V. M-DSR C.M-DSR Naive 26/26 $13 / 26 \quad 17 / 26 \quad 16 / 26 \quad \mathbf{2 2} / \mathbf{2 6}$

\section{General comments}

- Chinese Market is more volatile. The portfolio return has varied between $-4.17 \%$ and $4.56 \%$ during the test period. This variation was between $-1.98 \%$ and $1.47 \%$ for the American Market.

- For this two markets, all the methods perform better than the naive one.

- Conditional methods perform better than the non conditional ones. 
- The conditional Markowitz method is more interesting (in term of portfolio returns) than the classical Markowitz one.

- Similarly, the conditional Mean-DownSide risk is much more efficient than the classical Mean-DownSide optimization method.

\subsection{Another Tool to Test The Efficiency of the Proposed Methods}

The HangSeng and the S\&P 500 indices will contribute to test the efficiency of the classical and the new portfolio optimization methods. We will compare the daily portfolio return obtained by the weights which are solutions of different optimization programs using all the data until May, the 31th , 2015. From June, the 1st, 2015 until July, the 7th, 2015, we compute the portfolio returns and we compare them against the daily HangSeng and the S\&P 500 indices. Results are exhibited in figure 4.1 (Chinese Market) and figure 4.2 (American Market).

- Red color signifies a negative difference between portfolio return and HangSeng Index (respectively S\&P 500 index),

- blue color signifies a positive difference between portfolio return and HangSeng Index (respectively S\&P 500 index),

- Overall performance is the sum of all the differences during the test period.

We note that the comparison is in favour of the Conditional-Mean-Variance and the Conditional-Mean-Semivariance optimization methods. The overall performance varies depending on the method of portfolio optimization and markets :

- from $0.94 \%$ (Classical Markowitz method) to $12.64 \%$ (Conditional Mean-Semivariance method) for the Chinese Market,

- from $0.09 \%$ (Classical Markowitz method) to 1.42\% (Conditional Mean-Semivariance method) for the American Market.

These mouthwatering results deserve more practice and more tests over a long period of time and on different markets using different financial products. 

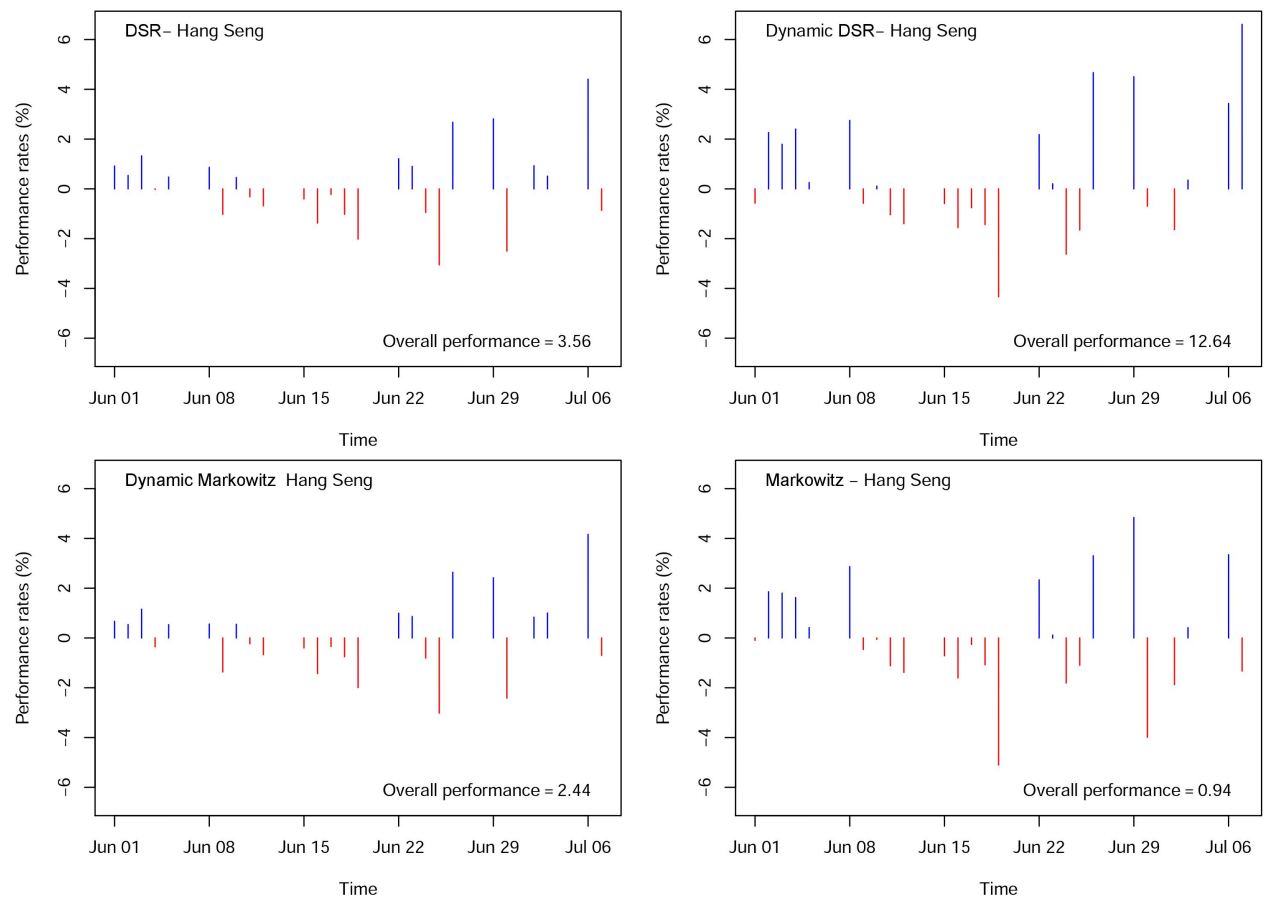

FIGURE 1 - HangSeng index versus return of optimal portfolio
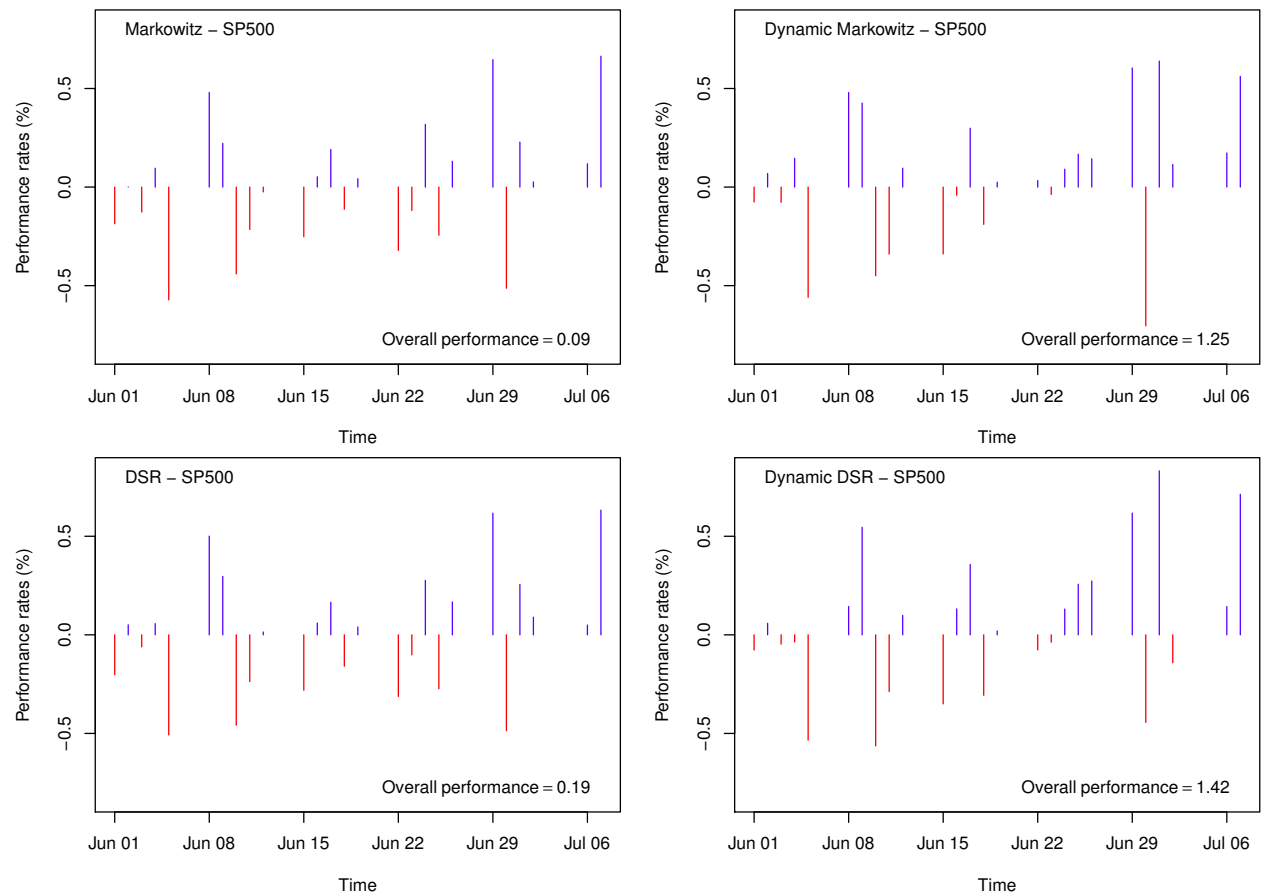

FIGURE 2 - S\&P500 index versus return of optimal portfolio 


\section{Conclusion}

In this paper, we developed two new approaches in order to get an optimal portfolio minimizing two conditional risks. The first risk is based on conditional variance, and the optimization method using this risk is an extension of the classical Mean-Variance model. Mean and Variance are replaced by Conditional Mean and Conditional Variance estimators. The second one is based on Conditional Semivariance, and the optimization method using this risk is an extension of the classical Mean-Semivariance model. This novelty, using conditional risk, gives a new approach and more efficient alternative to get an optimal portfolio. In fact, all the other methods do not anticipate the future and just extrapolate to the future what the observe in the past.

In both cases, the optimization algorithm involved using the Lagrangian method. Even, our results seem interesting, the efficiency of our methods should be confirmed on other markets and with other various assets. Kernel methods, belonging to nonparametric methods, are used to estimate Conditional Mean, Variance and Semivariance. Product Gaussian densities are used as kernel. It will be very helpful to choose typical multivariate kernels. Similarly, we should develop a global method to choose the bandwidth which is crucial in nonparametic estimation.

Back to results of this paper : the Conditional Mean-Semivariance seem most appropriate to get an optimal portfolio using the data of the Chinese and the American markets. By the way, Conditional Mean-Variance is more efficient than Mean-Variance method. Similarly, the Conditionam Mean-Semivariance is better than the Mean-Semivariance method. These conclusions are not definitive and should be confirmed or not under different hypothesis and on different markets.

Thanks to its robustness, it is also reasonable to substitute the conditional median to the conditional mean and to propose an optimization model based on Conditional Median and

conditional variance or conditional Median and Conditional Semivariance. This topic will be treated a matter for future research.

\section{Bibliography}

- Altman, N., Leger, C. (1995). Bandwidth Selection for Kernel Distribution Function Estimation, Journal of Statistical Planning and Inference,46, 195-214.

Arditti, F. D. (1971). Another Look at Mutual Fund Performance, Journal of Financial and Quantitative Analysis, 6, 3, 909-912.

- Athayde, G. (2001). Building a Mean-Downside Risk Portfolio Frontier, Developments in Forecast Combination and Portfolio Choice, John Wiley and Sons.

- Athayde, G. (2003). The mean-downside risk portfolio frontier : a non-parametric approach, Advances in portfolio construction and implementation.

- Ben Salah, H., Chaouch, M., Gannoun, A., de Peretti, C., Trabelsi, A. (2015). 
Median-based Nonparametric Estimation of Returns in Mean-Downside Risk Portfolio frontier, Manuscript submitted.

- Ben Salah, Gannoun, A., de Peretti, Ribatet, M., Trabelsi, A. (2015). A New Approach in Nonparametric Estimation of Returns in Mean-Downside Risk Portfolio frontier, Manuscript submitted.

- Black, F., Litterman, R. (1992). Global portfolio optimization, Financial Analysts Journal, 48,5, 28-43.

- Chunhachinda, P., Dandapani, K., Hamid, S., and Prakash, A.J. (1997). Portfolio Selection and Skewness : Evidence from International Stock Markets, Journal of Banking and Finance, 21, 143-167.

- Estrada, J. (2004). Mean-Semivariance Behavior : An Alternative Behavioural Model, Journal of Emerging Market Finance, 3, 231-248.

- Estrada, J. (2008). Optimization : A Heuristic Approach, Journal of Applied Finance, $57-72$.

- Harlow, V. (1991). Asset allocation in a downside risk framework, Financial Analyst Journal, 47, 28-40.

- Hogan, W. and Warren, J. (1974). Computation of the efficient Boundary in the ES Portfolio selection, Journal of Financial and Quantitative Analysis, 9, 1-11.

- Ledoit, O. and Wolf, M. (2004). Honey, I Shrunk the Sample Covariance Matrix, The Journal of Portfolio Management, 30, 4, 110-119.

- Levy, M., Roll, R. (2010). The Market Portfolio May Be Mean/Variance Efficient After All, Review of Financial Studies, 23, 6, 2464-2491.

- Li, Y., Hong, M., Tuner, N.D., Lupton, J.R, Caroll, R.J.. (2007). estimation of correlation functions in longitudinal and spatial data, with application to colon carcinogenesis experiments, J. Ann. Statist. 35, N, 1608-1643.

- Mamoghli, C. and Daboussi, S. (2008). Optimisation de portefeuille downside risk, Social Science Research Network, 23 pages.

- Markowitz, H. (1952). Portfolio Selection, Journal of Finance, 7, 77-91.

- Markowitz, H. (1959). Portfolio Selection : Efficient Diversification of Investments, New York : John Wiley \& Sons.

- Markowitz, H. (1987). Mean-Variance Analysis in Portfolio Choice and Capital Markets, Basil Blackwell, paperback edition, Basil Blackwell, 1990.

- Markowitz, H. , Todd, P., Xu, G., and Yamane, Y. (1993). Computation of meanSemivariance efficient sets by the critical line algorithm, Annals of Operations Research, 45, 307-317.

- Michaud, R. O. (1989). The Markowitz Optimization Enigma : Is 'Optimized' Optimal ?, Financial Analysts Journal , 45, 31-42.

Sarda, P. (1993). Smoothing parameter selection for smooth distribution functions, Journal of Statistical Planning and Inference, 35, 65-75.

- Prakash, A.J., Chang, C., Pactwa, T.E. (2003). Selecting a Portfolio with Skewness : Recent Evidence from US, European, and Latin American Equity Markets, Journal of Banking and Finance, 27, 1375-1390.

- Pratt, J., W. (1964). Risk Aversion in the Small and in the Large, Econometrica, $32,1,122-136$.

- Tobin, J. (1958). Estimation of Relationships for Limited Dependent Variables, Econometrica, 26,1, 24-36. 
- Vasant, D., Jarke, M., and Laartz, J. (2014). Big Data, Business \& Information Systems Engineering, 6,5, 257-259.

- Yin, J., Geng, Z., Wang,H. (2010). covariance model, Statistica Sinica, 20, 469-479. 